\title{
Paper
}

\section{An Improved Downlink MC-CDMA System for Efficient Image Transmission}

\author{
Mohammed Sofiane Bendelhoum ${ }^{1,2}$, Ali Djebbari², Ismail Boukli-Hacene $^{3}$, \\ and Abdelmalik Taleb-Ahmed ${ }^{4}$ \\ ${ }^{1}$ Department of Technologie, Sciences Institute, University Center Nour Bachir of El-Bayadh, Algeria \\ ${ }^{2}$ Telecommunications and Digital Signal Processing Laboratory, Djillali Liabes University, Algeria \\ ${ }^{3}$ Biomedical Laboratory, Abou Bekr Belkaid University, Tlemcen, Algeria \\ ${ }^{4}$ Laboratoire LAMIH UMR CNRS 8530, Valenciennes et du Hainaut Cambrésis University, Valenciennes, France
}

\begin{abstract}
Image compression is an essential stage of the data archiving and transmitting process, as it reduces the number of bits and the time required to complete the transmission. In this article, a study of image transmission over the Multi-Carrier Code Division Multiple Access (MC-CDMA) downlink system is presented. The solution proposed relies on source coding combined with channel coding. The Discrete Wavelet Transform (DWT) method is used in conjunction with the SPIHT coder to compress the image, then the data generated is transmitted with the MC-CDMA technique over a noisy channel. The results show that image transmissions performed over MC-CDMA using the SPIHT model are better than the traditional approach like MC-CDMA in the AWGN channel.
\end{abstract}

Keywords-data compression, MC-CDMA, MSSIM, performance evaluation, PSNR, SNR, SPIHT.

\section{Introduction}

One of the most important results of Shannon's work [1] was that source coding and channel coding can be treated separately without sacrificing fidelity [2]. Traditionally, the source and channel codes are designed separately and then cascaded together [3].

Multi-carrier modulation such as Orthogonal Frequency Division Multiplexing (OFDM) has a promising future as a new technology in next-generation wireless communication systems [4], [5]. Combining OFDM with Code Division Multiple Access (CDMA) results in the Multicarrier CDMA system [6], [7]. This system has received much attention among researchers. However, it suffers from Multiple Access Interference (MAI) in a multi-user setting, which decreases the overall Bit Error Rate (BER) performance [5]. Multi-user Detection (MUD) techniques have been introduced to mitigate MAI to improve the system performance [8], [9].

Several studies in transmission of images and multimedia using MC-CDMA technology are considered [5], [10], [11]. In [10], a study of real-time image traffic over a radio link using the wavelet transform (WT) to compress images and a CDMA link to transfer these images over wireless communication networks was presented. Images compressed with JPEG2000 are transmitted at low SNRs with the MC-CDMA technique and are considered in [11]. An efficient approach for transmitting images over MC-CDMA systems was examined in [5]. Furthermore, several coding techniques have been investigated for efficient transmission of images with MC-CDMA over wireless channels [10], [12], [13]. Despite the efficiency of these coding techniques, they add much redundancy to the transmitted data, which increases the bandwidth and reduces the channel utilization [5].

This paper studies an unage transmission efficiency scheme relying on the MC-CDMA system using discrete wavelet transforms (DWT) and set partitioning in hierarchical trees (SPIHT) coding in the Additive White Gaussian Noise (AWGN) channel. The results are compared with the traditional MC-CDMA technique. In the simulation conducted, the images are issued with the use of both techniques, and their reception is objectively evaluated by means of PSNR and MSSIM parameters. The transmission of images is done with a different SNR.

The remainder of the paper is organized as follows: in Section 2, a description of decomposition of the image using DWT is presented. Section 3 exposes the SPIHT encoder. The parameters used for judging the quality of images are introduced in Section 4. In Section 5 the MC-CDMA model used for transmission and reception is given. Section 6 presents experimental and performance results. Finally, Section 7 offers conclusions and remarks.

\section{Wavelet Transform}

The emergence of wavelets has led to the convergence of linear expansion methods used in signal processing and applied mathematics. Subband coding methods and their associated filters are closely related to the wavelet concept [13]. 
A wavelet is a foundation for representing images in various degrees of resolution. Wavelet transform is a mathematical function dividing an image into two various frequency components matching the resolution. The wavelet transformation methodology has been used because of the disadvantages of Fourier transformation [14]. Wavelet transformation has been classified, as mentioned earlier, as DWT and continuous wavelet transforms (CWT). A wavelet is represented as a multi-resolution level, where each analysis is implemented through high-pass and low-pass filters, where each high-pass filter is based on wavelets and low-pass filter is based on scaling functions. The wavelet transform function is based on the conversion of a one-dimensional function into two-dimensional spaces involving translation and dilation parameters related to time and scale factors [15].

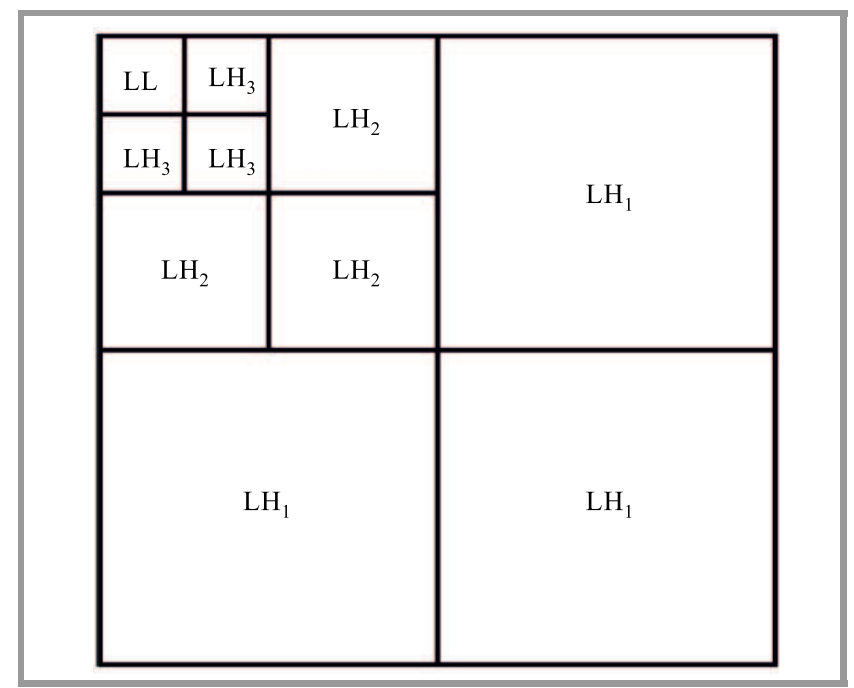

Fig. 1. Pyramidal structure of 3-level wavelet decomposition.

Wavelet analysis of an image can be viewed in the frequency domain as partitioning it into a set of subbands, where each partitioning step is obtained by applying the $2 \mathrm{D}$ wavelet transform. One level of 2D wavelet transforms results in four sets of data (wavelet coefficients) that correspond to four $2 \mathrm{D}$ frequency subbands. For these four subbands, if the original image data is on the zerodecomposition level (scale), we use the following notation on the k-th decomposition level: HHk (high-high or diagonal details), HLk (high-low or horizontal details), LHk (low-high or vertical details), LLk (low-low or approximation). The LLk subband is also referred to as image approximation, as it represents the image on a lower scale, while to other subbands are referred as to image details. Wavelet decomposition is dyadic when only the LLk subband is further transformed. It results in a new set of subbands: $\mathrm{HHk}+1, \mathrm{HLk}+1, \mathrm{LHk}+1, \mathrm{LLk}+1$. Dyadic decomposition used in image compression will thus generate a hierarchical pyramidal structure, as shown in Fig. 1. If the dyadic decomposition of $\mathrm{N}$ levels is performed (low-low subband transformation performed $\mathrm{N}$ times), the result will be $3 \mathrm{~N}+1$ subbands [16].

\section{SPIHT Coding Scheme}

In 1996, Said and Pearlman introduced the SPIHT coding scheme [17]. SPIHT is viewed as one of the most efficient wavelet-based image compression algorithms available in the literature [17], [18]. It can be a progressive transmission and it may be relevant for the lossless compression area of interest. It takes advantage of the spatial orientation of the zerotree coding that was proposed by Shapiro [19] and has been shown to provide better performance [20]. In addition, SPIHT can compress images in a lossless manner, as well as with a loss [21].

SPIHT [22] is one of the most advanced schemes available, even outperforming the state-of-the-art JPEG 2000 in some situations. The basic principle is the same - progressive coding is applied, processing the image respectively to a lowering threshold. The difference is in the concept of zerotree (spatial orientation trees in SPIHT).

This is an idea that takes into consideration relations between coefficients across subbands at different levels [17]. The first idea is always the same. If there is a coefficient at the highest level of the transform in a particular subband which is considered insignificant against a particular threshold, it is very probable that its descendants in lower levels will be insignificant too. Therefore, we can code quite a large group of coefficients with one symbol.

Figure 2 shows how a spatial orientation tree is defined in a pyramid constructed with recursive four subbands splitting. The coefficients are ordered hierarchically. According to this relationship, the SPIHT algorithm saves many bits that specify insignificant coefficients [23].

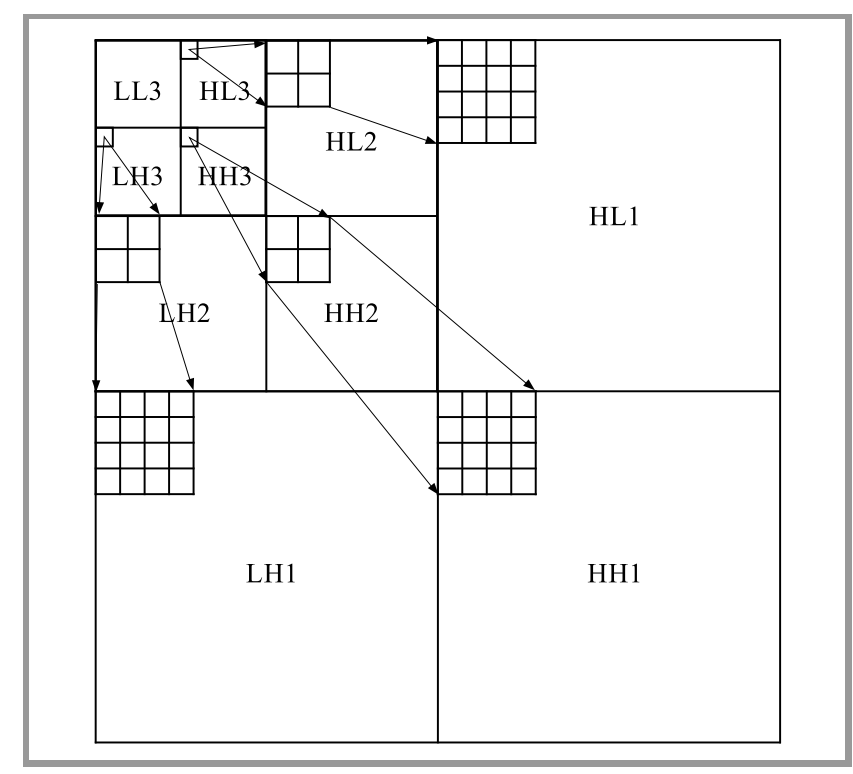

Fig. 2. Parent-child relationship.

The SPIHT flowchart is presented in Fig. 3. In the first step, the original image is decomposed into subbands. Then, the method finds the maximum and the iteration number. In the second step, the method puts the wavelet coefficients into a sorting pass that finds all significant values and encodes 
their sign. In the third step, the significant coefficients that were found in the sorting pass are put into the refinement pass that uses two bits to exactly reconstruct the value for closing to the real value [24].

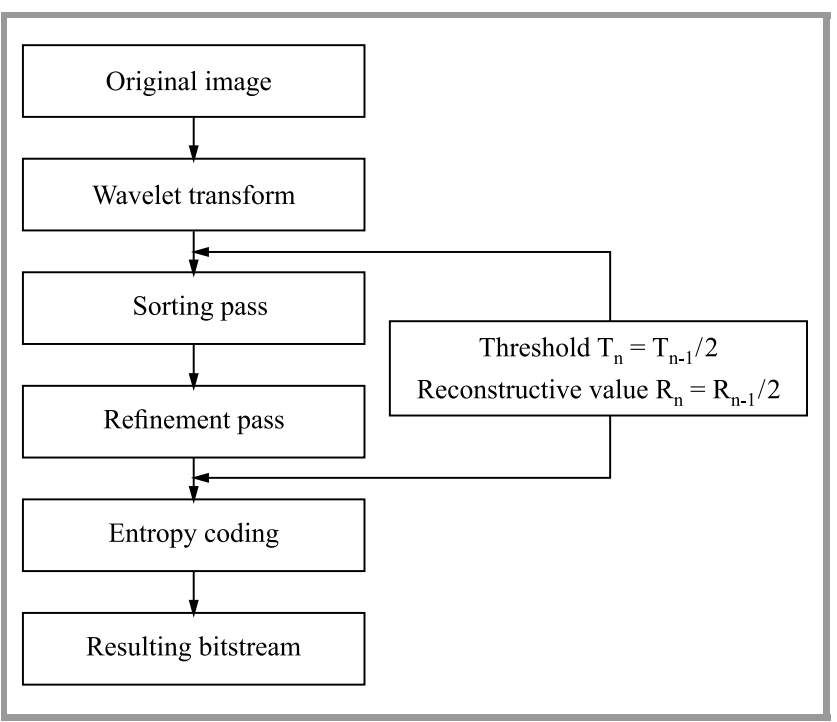

Fig. 3. Flowchart of SPIHT wavelet transform.

\section{Quality Evaluation}

The peak signal to noise ratio (PSNR) is the most commonly used reconstruction quality measure in image compression:

$$
\text { PSNR }=10 \log \frac{(\text { Dynamics of image })^{2}}{M S E} .
$$

Mean square error (MSE) which requires two $M \times N$ gray scale images $I$ and $\hat{I}$ where one of the images is considered as a compression of the

$$
\mathrm{MSE}^{2}=\frac{1}{M \times N} \sum_{i=1}^{N} \sum_{j=1}^{M}(I(i, j)-\hat{I}(i, j))^{2} .
$$

Usually an image is encoded by 8 bits. It is represented by 256 gray levels, which vary between 0 and 255 , with the extent or dynamics of the image being 255 .

The PSNR measurement gives a numerical value on the damage, but it does not describe its type. Moreover, as is often noted in [25], [26], it does not quite represent the quality perceived by human observers. For different applications where images that are degraded must eventually be examined by experts, traditional evaluation remains insufficient. For this reason, objective approaches are needed to assess the image quality. Then, we evaluate a new paradigm to estimate the quality of images, specifically the ones compressed by wavelet transform based on the assumption that the human visual system (HVS) is highly adapted to extract structural information. The similarity compares the brightness, contrast and structure between each pair of vectors, where the structural similarity index (SSIM) between two signals $x$ and $y$ is given by [27], [28]:

$$
\operatorname{SSIM}(x, y)=l(x, y) c(x, y) s(x, y) .
$$

Finally, the quality measurement can provide a spatial map of the local image quality, which gives more information on the image quality degradation. For application, we require a single overall measurement of the whole image quality that is given by the following formula:

$$
\operatorname{MSSIM}(I, \hat{I})=\frac{1}{M} \sum_{i=1}^{M} \operatorname{SSIM}(I, \hat{I}),
$$

where $I$ and $\hat{I}$ are respectively the reference and degraded images respectively, $I_{i}$ and $\hat{I}_{i}$ are the contents of images at the $i$-th local window. $M$ is the total number of local windows in the image. The MSSIM values exhibit greater consistency with the visual quality.

\section{Model MC-CDMA System}

In general, there are three types of hybrid schemes, e.g. Multi-Carrier Direct Sequence CDMA (MC/DS-CDMA), Multicarrier-CDMA (MC-CDMA) and Orthogonal Frequency Code Division Multiplexing (OFCDM) [29]. The basic MC-CDMA signal is generated by a serial concatenation of classical Direct-Sequence CDMA (DS-CDMA) and Orthogonal Frequency Division Multiplexing (OFDM). Each chip of the direct sequence spread data symbol is mapped to a different subcarrier. Thus, with MC-CDMA, the chips of a spread data symbol are transmitted in parallel on different sub-carriers, in contrast to serial transmission with DS-CDMA [5], [30]. The MC-CDMA system model using for transmission data image is divided into four stages, including the image data compression stage using DWT and SPHIT, the BPSK modulation stage, the spreading stage using the Walsh code, and the OFDM modulation stage, as shown in Fig. 4.

At the transmitter, the bit stream generated after compression step $b_{i}^{(k)}$ is modulated by BPSK $B_{i}^{(k)}$. The BPSK modulated information bits of each user $k=0, \ldots, k-1$, are spread by the corresponding spreading code, where WalshHadamard codes are applied. In the downlink system, the spread data of all active users are added synchronously and mapped on to the $L_{c}$ sub-carriers [7]. The sequence, $B_{i}^{(k)}$ is applied to a serial-to-parallel converter and multiplied by the $k$ with the user specific spreading code of Walsh $C_{i}^{(k)}$ of length $L$ [5], [7], In [31], [7] confirmed that the WalshHadamard codes are the best choice for the downlink, also for the minimization of multiple access interference.

$$
C_{i}^{(k)}=\left(C_{0}^{(k)}, C_{1}^{(k)}, \ldots, C_{L-1}^{(k)}\right)^{T}
$$

The chip rate of the serial spreading code of Walsh $C_{i}^{(k)}$ before serial-to-parallel conversion is [7]:

$$
\frac{1}{T_{c}}=\frac{L}{T_{B}} \text {. }
$$


(a)

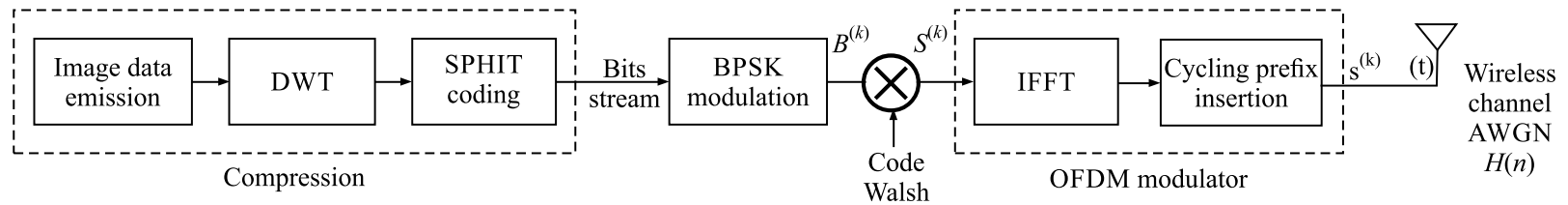

(b)

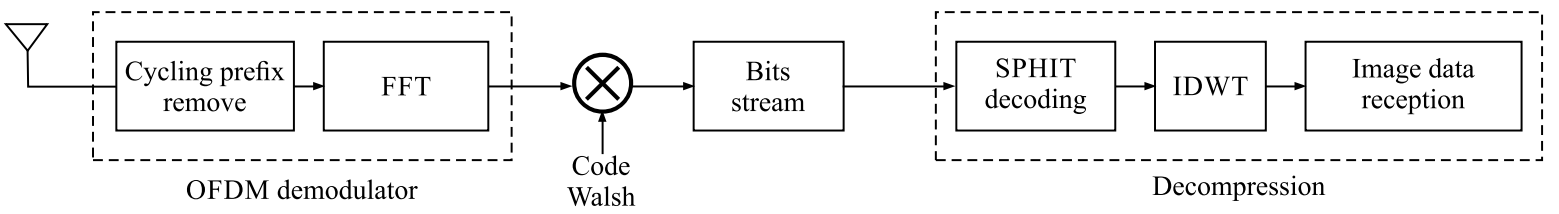

Fig. 4. The MC-CDMA system model for image transmission: (a) transmitter, (b) receiver.

The chip rate is $L$ times higher than the data symbol rate $\frac{1}{T_{B}}$. The complex valued sequence obtained after spreading can be expressed in a vector notation as [7]:

$$
s_{i}^{(k)}=B_{i}^{(k)} \cdot C_{i}^{(k)}=\left(s_{0}^{(k)}, s_{1}^{(k)}, \ldots, S_{L-1}^{(k)}\right)^{T} .
$$

Then $s_{i}^{(k)}$ is modulated by the OFDM modulator, the input signal of the OFDM operation is statistically independent [7]. The result is $S_{i}^{(k)}$. The advantage of the frequency domain channel model is that the IFFT and FFT operation for OFDM and inverse OFDM can be avoided, and the fading operation results in one complex-valued multiplication per subcarrier [7]. The spectral containment of the channels is better since they are not using cyclic prefix [32] and it reduces the Inter-Symbol Interference (ISI) effect.

Signal $H$ is obtained by adding the $S_{i}^{(k)}$ from $K$ users:

$$
H=\sum_{1=0}^{k} S_{i}^{(k)} .
$$

The result $H$ is applied to a parallel-to-serial converter. The transmitted vector $T_{X}$ is given by:

$$
T_{X}(t)=H_{c}+n
$$

where $H_{c}$ is the data channel vector and $n$ is the noise AWGN vector.

The steps used to transmit data images using the MCCDMA model can be summarized as follows:

1. The data image is transformed by DWT in the same dimension as the original image.

2. The result of the step 1 is associated with SPIHT coding to generate the bits stream to be transmitted also at a different bit rate of $R_{c}$.

3. It is assumed that all subchannels are identically modulated using binary phase-shift keying (BPSK) [33].

4. The most important characteristic of the sequence in the MC-CDMA system is the zero-cross correlation property. The most widely used orthogonal code is the Walsh-Hadamard code [12], which is generated by using the Hadamard transform defined as:

$$
\begin{gathered}
C_{0}=[0], \\
C_{2 m}=\left[\begin{array}{ll}
C_{m} & C_{m} \\
C_{m} & \overline{C_{m}}
\end{array}\right] .
\end{gathered}
$$

This transform gives matrix, $C_{m}$, for $m=2^{i}$ matrix, where $i$ is an integer. For example, consider a case of $n=4$ :

$$
C_{4}=\left[\begin{array}{ll}
C_{2 m} & C_{2 m} \\
C_{2 m} & C_{2 m}
\end{array}\right]=\left[\begin{array}{llll}
0 & 0 & 0 & 0 \\
0 & 1 & 0 & 1 \\
0 & 0 & 1 & 1 \\
0 & 1 & 1 & 0
\end{array}\right] .
$$

5. An OFDM modulation step is performed on the binary data.

6. At the receiver, after OFDM demodulation step. The vector is multiplied by the $C_{i}^{(k)}$ code Walsh for $k$-th user, and we applied the SPIHT decoding method. The inverse discrete wavelet transform (IDWT) is used to receive the data image.

Finally, the decompressed image is given at the transmitted bit rate of $R_{c}$.

\section{Results and Discussion}

After various applications intended to show the actual performance of the method proposed, we will make a comparison between the following, different types of wavelet trans-

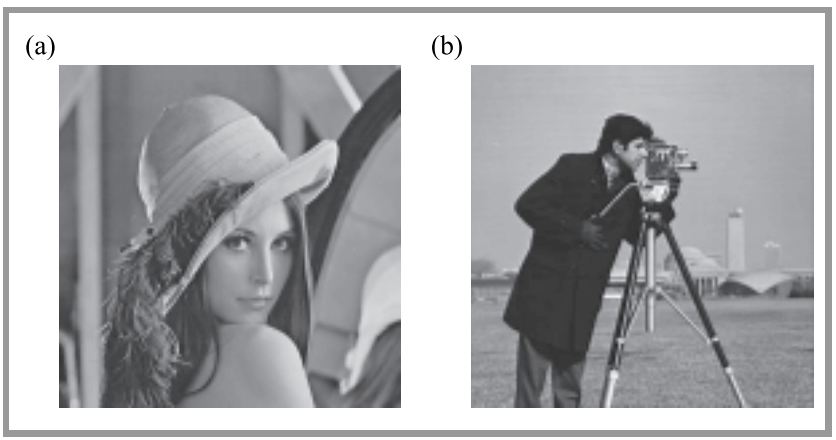

Fig. 5. Typical test pictures: (a) Lena, (b) cameraman. 
forms: Daubechies (db5), symelets (sym9), Coiflets (coif5), Bi-orthogonal (bior4.4) and Cohen-Daubechies-Feauveau (CDF9/7). They are associated with an SPIHT coder in the Subsection 6.1. The Lena and Cameraman images (Fig. 5) with $128 \times 128$ pixels (grayscale) are encoded $(8$ bits per pixel) for each application. In the step compression step, we vary the bitrate from 0.125 to 1 and transmit them via the MC-CDMA model, at different SNRs - from 5 to $20 \mathrm{~dB}$ - in 5 steps. The quality of the images transmitted and received is evaluated objectively with PSNR and MSSIM parameters for different bit rates $R_{c}$.

Subsection 6.3 gives the performance of the MC-CDMA system in the AWGN channel with multiple users. The simulation values are depicted in a graph/plot of BER vs. $\mathrm{Eb} / \mathrm{NO}$ (in $\mathrm{dB}$ ), which is the energy per bit per noise spectral density [12], [13]. It defines the SNR per bit and it is an important measurement to evaluate and compare different digital communication systems.

The CDF9/7 wavelet is implemented with a SPIHT coder. Tree $128 \times 128$ images are used: IRM1, IRM2 and IRM3. The original image is compressed with different bit rates $R_{c}$ and transmitted at different SNRs for 10 to $17 \mathrm{~dB}$ in $1 \mathrm{~dB}$ steps. The image transmitted and reconstructed at different SNRs is estimated with PSNR and MSSIM factors.

In calculations and numerical simulations, the same images as those treated in [5] are used to evaluate the performance of the simulation model presented.

\subsection{Wavelets Association with SPIHT in MC-CDMA}

The average PSNR and MSSIM values for the Lena image at different $R_{c}$ are shown in Tables 1-5, enabling the judgment of quality of the SPIHT compressed image after transmission, as compared to its quality before transmission.

A plot of the variation of PSNR and MSSIM for the Lena image with different SNRs at $R_{c}$ constant is shown in Figs. 6-7.

Table 1

PSNR and MSSIM values for the Lena image at different $R_{c}$ and SNR with bior4.4 and SPIHT

\begin{tabular}{|c|c|c|c|c|c|c|}
\hline \multirow{2}{*}{$\begin{array}{l}\text { Parameter } \\
\text { of } \\
\text { evaluation }\end{array}$} & \multirow{2}{*}{$\begin{array}{c}R_{c} \\
{[\mathrm{bpp}]}\end{array}$} & \multirow{2}{*}{$\begin{array}{l}\text { Before } \\
\text { trans- } \\
\text { mission }\end{array}$} & \multicolumn{4}{|c|}{$\begin{array}{l}\text { Transmission with SNR } \\
{[\mathrm{dB}]}\end{array}$} \\
\hline & & & 5 & 10 & 15 & 20 \\
\hline PSNR [dB] & \multirow{2}{*}{0.125} & 30.54 & 27.56 & 27.70 & 30.47 & 30.54 \\
\hline MSSIM & & 0.662 & 0.076 & 0.164 & 0.661 & 0.662 \\
\hline PSNR [dB] & \multirow{2}{*}{0.25} & 31.19 & 27.51 & 28.59 & 29.03 & 31.19 \\
\hline MSSIM & & 0.749 & 0.066 & 0.117 & 0.405 & 0.749 \\
\hline PSNR [dB] & \multirow{2}{*}{0.5} & 32.53 & 28.01 & 27.70 & 29.83 & 32.53 \\
\hline MSSIM & & 0.840 & 0.081 & 0.138 & 0.557 & 0.840 \\
\hline PSNR [dB] & \multirow{2}{*}{0.75} & 34.35 & 27.36 & 28.03 & 29.65 & 34.35 \\
\hline MSSIM & & 0.899 & 0.059 & 0.214 & 0.537 & 0.899 \\
\hline PSNR [dB] & \multirow[t]{2}{*}{1} & 35.76 & 28.91 & 27.76 & 29.09 & 35.76 \\
\hline MSSIM & & 0.927 & 0.028 & 0.177 & 0.415 & 0.927 \\
\hline
\end{tabular}

Table 2

PSNR and MSSIM values for the Lena image at different $R_{c}$ and SNR with coif2 and SPIHT

\begin{tabular}{|c|c|c|c|c|c|c|}
\hline \multirow{2}{*}{$\begin{array}{c}\text { Parameter } \\
\text { of } \\
\text { evaluation }\end{array}$} & \multirow{2}{*}{$\begin{array}{c}R_{c} \\
{[\mathrm{bpp}]}\end{array}$} & \multirow{2}{*}{$\begin{array}{l}\text { Before } \\
\text { trans- } \\
\text { mission }\end{array}$} & \multicolumn{4}{|c|}{$\begin{array}{c}\text { Transmission with SNR } \\
{[\mathrm{dB}]}\end{array}$} \\
\hline & & & 5 & 10 & 15 & 20 \\
\hline PSNR [dB] & \multirow{2}{*}{0.125} & 30.48 & 28.76 & 29.04 & 30.27 & 30.48 \\
\hline MSSIM & & 0.663 & 0.095 & 0.114 & 0.646 & 0.663 \\
\hline PSNR [dB] & \multirow{2}{*}{0.25} & 32 & 29.19 & 30.70 & 31.17 & 31.32 \\
\hline MSSIM & & 0.753 & 0.070 & 0.126 & 0.746 & 0.753 \\
\hline PSNR [dB] & \multirow{2}{*}{0.5} & 32.63 & 29.11 & 29.24 & 31.40 & 32.63 \\
\hline MSSIM & & 0.849 & 0.120 & 0.121 & 0.743 & 0.849 \\
\hline PSNR [dB] & \multirow{2}{*}{0.75} & 34.44 & 29.15 & 29.55 & 31.68 & 34.44 \\
\hline MSSIM & & 0.902 & 0.133 & 0.138 & 0.520 & 0.902 \\
\hline PSNR [dB] & & 35.90 & 29.16 & 29.60 & 32.55 & 35.90 \\
\hline MSSIM & & 0.928 & 0.135 & 0.135 & 0.827 & 0.928 \\
\hline
\end{tabular}

Table 3

PSNR and MSSIM values for the Lena image at different $R_{c}$ and SNR with with sym9 and SPIHT

\begin{tabular}{|c|c|c|c|c|c|c|}
\hline \multirow{2}{*}{$\begin{array}{c}\text { Parameter } \\
\text { of } \\
\text { evaluation }\end{array}$} & \multirow{2}{*}{$\begin{array}{c}R_{c} \\
{[\mathrm{bpp}]}\end{array}$} & \multirow{2}{*}{$\begin{array}{l}\text { Before } \\
\text { trans- } \\
\text { mission }\end{array}$} & \multicolumn{4}{|c|}{$\begin{array}{l}\text { Transmission with SNR } \\
{[\mathrm{dB}]}\end{array}$} \\
\hline & & & 5 & 10 & 15 & 20 \\
\hline PSNR [dB] & \multirow{2}{*}{0.125} & 30.34 & 28.30 & 28.48 & 28.94 & 30.34 \\
\hline MSSIM & & 0.655 & 0.030 & 0.149 & 0.408 & 0.655 \\
\hline PSNR [dB] & \multirow{2}{*}{0.25} & 31.25 & 28.40 & 28.52 & 30.16 & 31.25 \\
\hline MSSIM & & 0.750 & 0.039 & 0.152 & 0.635 & 0.750 \\
\hline PSNR [dB] & \multirow{2}{*}{0.5} & 32.69 & 28.48 & 28.59 & 29.52 & 32.69 \\
\hline MSSIM & & 0.847 & 0.053 & 0.138 & 0.501 & 0.847 \\
\hline PSNR [dB] & \multirow{2}{*}{0.75} & 34.56 & 28.05 & 28.10 & 28.82 & 34.56 \\
\hline MSSIM & & 0.903 & 0.054 & 0.142 & 0.327 & 0.903 \\
\hline PSNR [dB] & \multirow{2}{*}{1} & 36.02 & 28.58 & 29.00 & 31.22 & 36.02 \\
\hline MSSIM & & 0.938 & 0.079 & 0.143 & 0.761 & 0.938 \\
\hline
\end{tabular}

Table 4

PSNR and MSSIM values for the Lena image at different $R_{c}$ and SNR with bior4.4 and SPIHT

\begin{tabular}{|c|c|c|c|c|c|c|}
\hline \multirow{2}{*}{$\begin{array}{l}\text { Parameter } \\
\text { of } \\
\text { evaluation }\end{array}$} & \multirow{2}{*}{$\begin{array}{c}R_{c} \\
{[\mathrm{bpp}]}\end{array}$} & \multirow{2}{*}{$\begin{array}{l}\text { Before } \\
\text { trans- } \\
\text { mission }\end{array}$} & \multicolumn{4}{|c|}{$\begin{array}{l}\text { Transmission with SNR } \\
{[\mathrm{dB}]}\end{array}$} \\
\hline & & & 5 & 10 & 15 & 20 \\
\hline PSNR [dB] & \multirow{2}{*}{0.125} & 30.54 & 27.56 & 28.38 & 29.54 & 30.54 \\
\hline MSSIM & & 0.672 & 0.076 & 0.185 & 0.661 & 0.672 \\
\hline PSNR [dB] & \multirow{2}{*}{0.25} & 31.35 & 28.16 & 29.27 & 30.52 & 31.35 \\
\hline MSSIM & & 0.766 & 0.037 & 0.120 & 0.690 & 0.766 \\
\hline PSNR [dB] & \multirow{2}{*}{0.5} & 32.85 & 28.69 & 27.87 & 30.77 & 32.85 \\
\hline MSSIM & & 0.858 & 0.050 & 0.206 & 0.695 & 0.858 \\
\hline PSNR [dB] & \multirow{2}{*}{0.75} & 34.74 & 27.25 & 29.73 & 30.54 & 34.74 \\
\hline MSSIM & & 0.911 & 0.042 & 0.087 & 0.650 & 0.911 \\
\hline PSNR [dB] & \multirow{2}{*}{1} & 36.37 & 27.87 & 28.37 & 30.29 & 36.37 \\
\hline MSSIM & & 0.939 & 0.040 & 0.134 & 0.664 & 0.939 \\
\hline
\end{tabular}


Table 5

PSNR and MSSIM values for Lena image at different $R_{c}$ and SNR with CDF9/7 and SPIHT

\begin{tabular}{|c|c|c|c|c|c|c|}
\hline \multirow{2}{*}{$\begin{array}{l}\text { Parameter } \\
\text { of } \\
\text { evaluation }\end{array}$} & \multirow{2}{*}{$\begin{array}{c}R_{c} \\
{[\mathrm{bpp}]}\end{array}$} & \multirow{2}{*}{$\begin{array}{c}\text { Before } \\
\text { trans- } \\
\text { mission }\end{array}$} & \multicolumn{4}{|c|}{$\begin{array}{c}\text { Transmission with SNR } \\
{[\mathrm{dB}]}\end{array}$} \\
\hline & & & 5 & 10 & 15 & 20 \\
\hline PSNR [dB] & \multirow{2}{*}{125} & 30.44 & 28.12 & 28.98 & 9 & 0.4 \\
\hline MSSIM & & 0.680 & 0.032 & 0.063 & 0.655 & 0.680 \\
\hline PSNR [dB] & \multirow{2}{*}{0.25} & 31.59 & 27.52 & 27.94 & 31.10 & 31.59 \\
\hline MSSIM & & 0.783 & 0.072 & 0.108 & 0.764 & 0.783 \\
\hline PSNR [dB] & \multirow{2}{*}{0.5} & 32.96 & 27.14 & 28.25 & 30.14 & 32.96 \\
\hline MS & & 0 & 7 & 31 & 0.631 & 0.870 \\
\hline PSNR [dB] & \multirow{2}{*}{0.75} & 35.19 & 27.44 & 27.81 & 31.29 & 35.19 \\
\hline MSSIM & & 0.917 & 0.073 & 0.093 & 0.738 & 0.917 \\
\hline PSNR [dB] & & 36 & 27.85 & 29.27 & 29.51 & 36.92 \\
\hline MSSIM & & 0.944 & 0.050 & 0.073 & 0.480 & 0.944 \\
\hline
\end{tabular}

For all figures the variations of PSNR and MSSIM, increases proportionally with the SNR. For the lower SNR $(10 \mathrm{~dB})$ the images that are received are of very poor qual- ity (low MSSIM). In the case of SNR greater than $10 \mathrm{~dB}$, the quality of images that are received increases until they reach the maximum value obtained without a transmission $(\mathrm{SNR}=20 \mathrm{~dB})$.

As far as the association of different types of wavelets (db5, coif2, sym9, bior4.4 and CDF9/7) with the SPHIT encoder at different bit rates (Rc) is concerned, let's say that the CDF9/7 wavelet provides the most important PSNR and MSSIM values compared to those obtained by other wavelets for a $\mathrm{SNR}=20 \mathrm{~dB}$ [20] (except for $R_{c}=0.125 \mathrm{bpp}$, where PSNR $=30.44 \mathrm{~dB}$ ).

The CDF9/7 wavelet will be subsequently retained for various images with different $R_{c}$ transmission in a telecommunications MC-CDMA system. The received images will be evaluated objectively by PSNR and MSSIM.

\subsection{Analysis of MC-CDMA Performance in a Wireless Channel}

Figure 8 shows the BER performance comparison for multiuser cases in the AWGN channel with the MC-CDMA model used and traditional MC-CDMA. In theory, BER lowers with increasing Eb/N0. It is observed that BER performance decreases as Eb/N0 increases. This is because

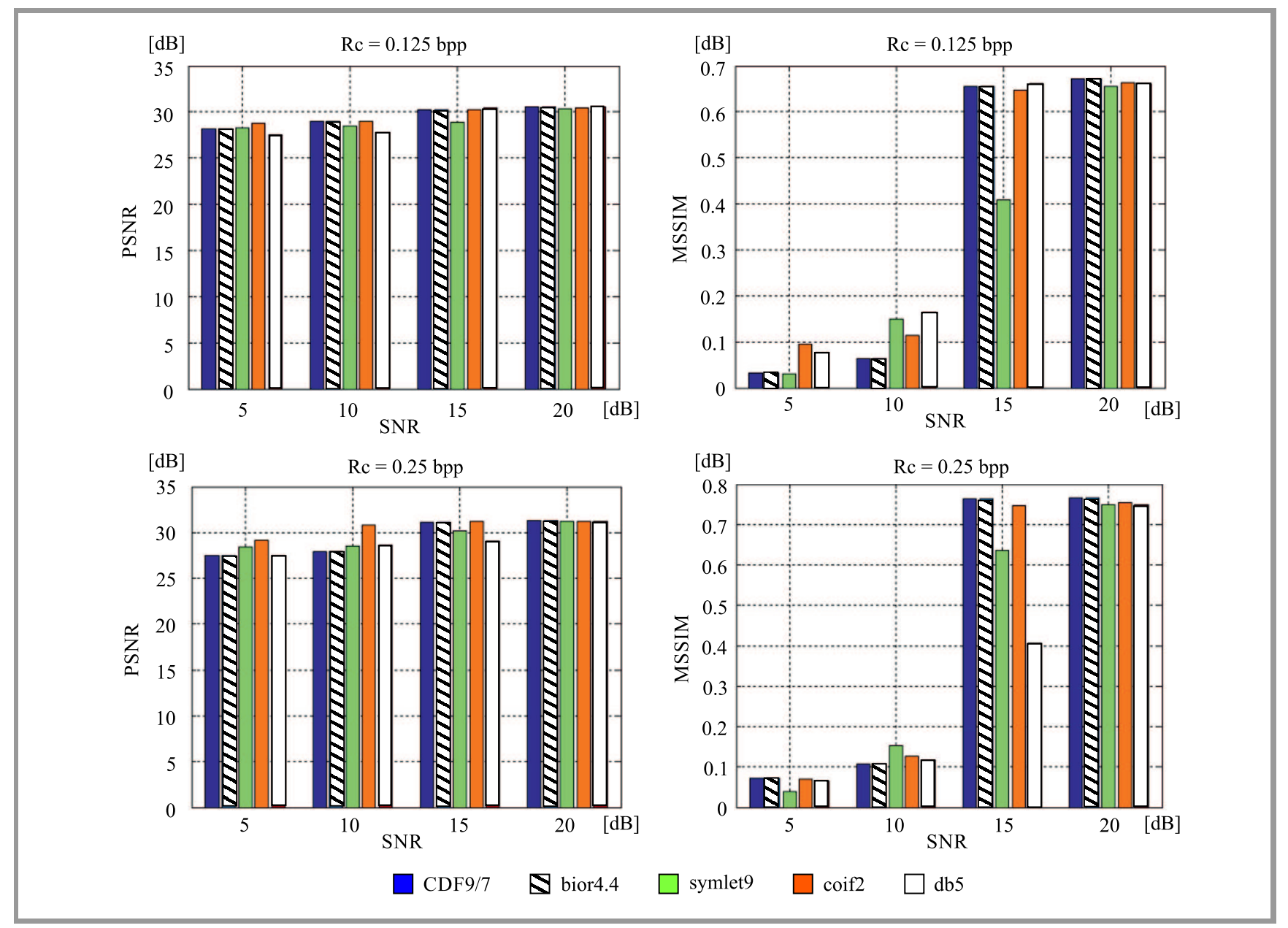

Fig. 6. Variation of PSNR and MSSIM for the Lena image with 0.125 and 0.25 bpp. (See color pictures online at www.nit.eu/ publications/journal-jtit) 

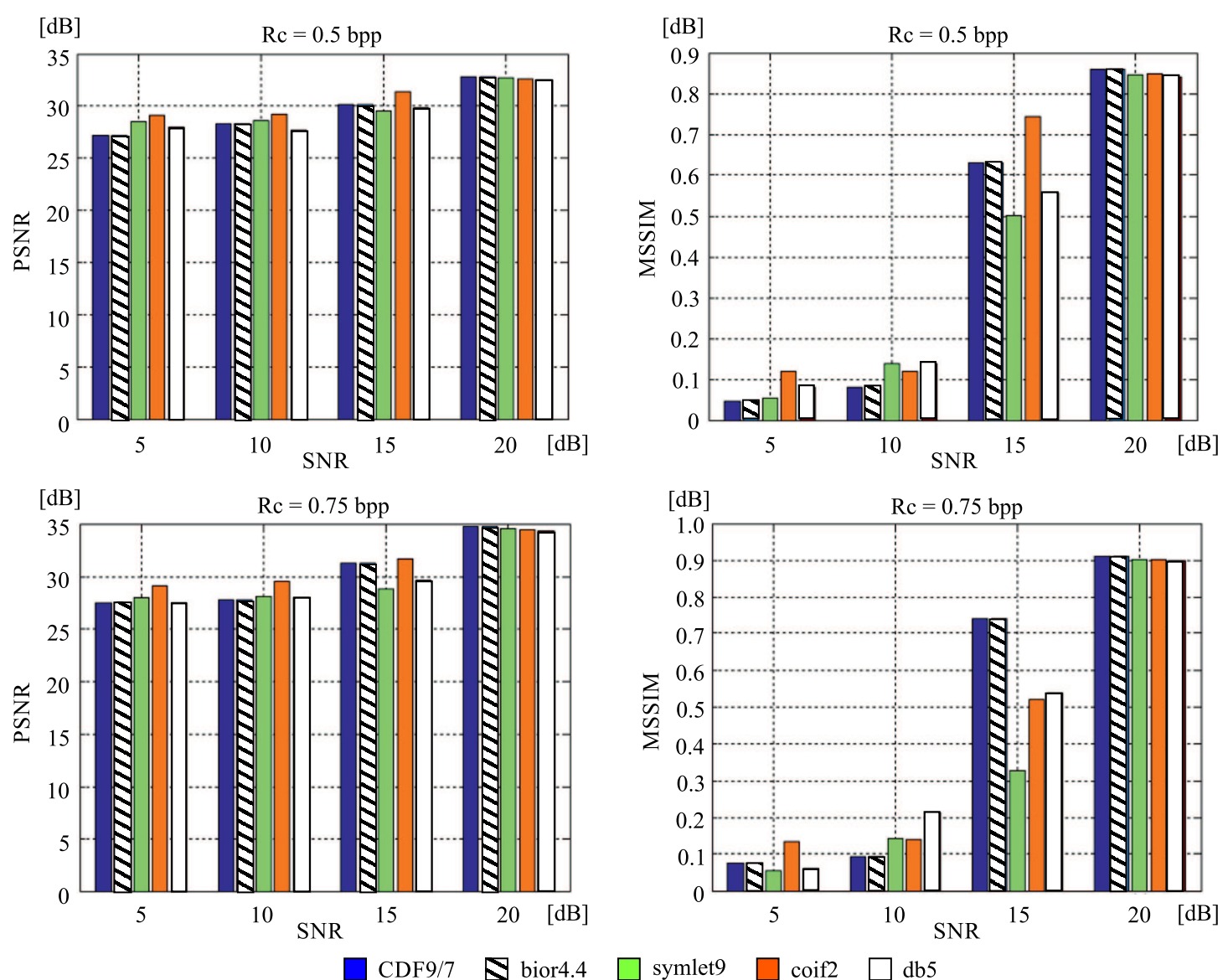

Fig. 7. Variation of PSNR and MSSIM for the Lena image with 0.5 and 0.75 bpp.

a lower AWGN noise level can be obtained with a higher $\mathrm{Eb} / \mathrm{NO}$. This is due to the fact that BER performance is only affected by AWGN noise [12], [13].

In the case of a low SNR, the two transmission systems show the same performance. As SNR increases, as it is clear than this transmission scheme offers better performance in terms of BER terminology. The BER results show that the proposed transmission scheme is based on the SPHIT coder coupled to MC-CDMA, and is superior to that obtained from the traditional MC-CDMA.

In the MC-CDMA model proposed the SNR increases to $15 \mathrm{~dB}$, in comparison with the traditional MC-CDMA technique, where the SNR reaches $22 \mathrm{~dB}$, which means a gain of about $7 \mathrm{~dB}$.

\subsection{Performance Analysis of CDF9/7 with SPIHT coder MC-CDMA System}

Several experiments were performed to test the performance and the robustness of the medical images transmitted through the MC-CDMA system using the CDF9/7 and SPHIT coder. The results of the simulation are presented in Tables 6-8 for all image users (IRM1, IRM2 and IRM3).
The IRM1 image transmission via a wireless MC-CDMA system with the image compressed at 0.125 bpp needs the $\mathrm{SNR}=15 \mathrm{~dB}$ to achieve the maximum PSNR and MSSIM values before the transmission. On the other hand, for the $R_{c}=0.25,0.5,0.75$ and $1 \mathrm{bpp}$ we need the SNR $=16 \mathrm{~dB}$ for a perfect transmission in our MC-CDMA system. The same remarks are noted in the case of IRM3 images.

For the transmission of the IRM2 image with $R_{c}=$ $0.125 \mathrm{bpp}$ and 0.25 , the $\mathrm{SNR}=15 \mathrm{~dB}$ is necessary, but for $R_{c}$ bpp greater than 0.25 all results show that the $\mathrm{SNR}=16 \mathrm{~dB}$ can perfectly transmit all images.

Examples of the received images are shown in Figs. 9-11for $\mathrm{SNR}=14,15$ and $16 \mathrm{~dB}$.

Using human eyesight only, we can see that the images recovered are not identical with the originals, once the SNR is lowered to $14 \mathrm{~dB}$. One can observe that the best quality is obtained at $\mathrm{SNR}=16 \mathrm{~dB}$, in this case the MC-CDMA model is considered as a reliable wireless communications system. The PSNR of the received images are exceeds $30 \mathrm{~dB}$, which means that we can also transmit an image using $R_{c}=0.75 \mathrm{bpp}$ and obtain a good level of visual quality (MSSIM $\geq 0.8$ ) of the received image.

All results obtained by transmitting the images over the MC-CDMA model are better than those relying on the traditional MC-CDMA technique. 

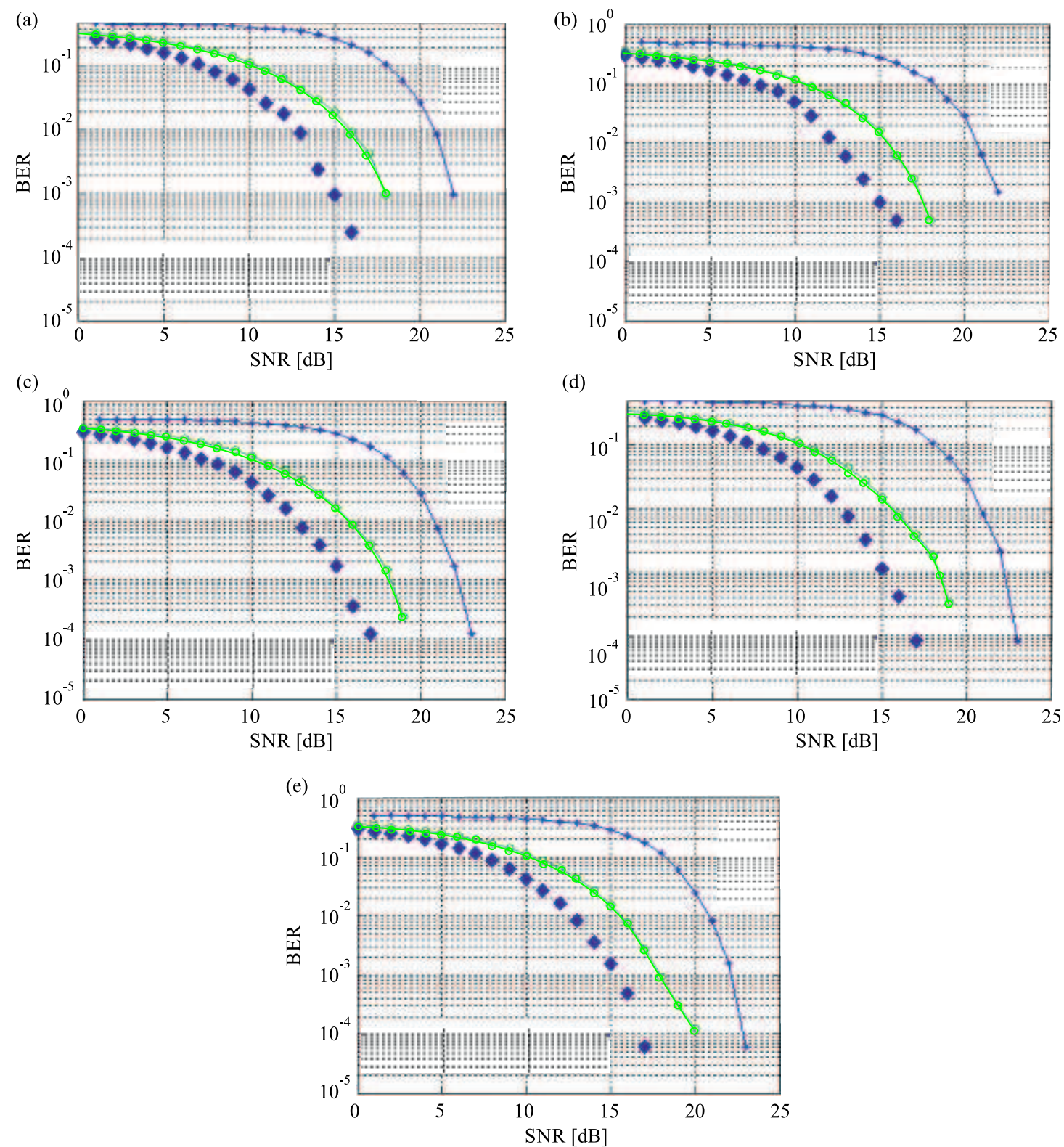

$\checkmark$ simulated BER for Lena $\quad \_$simulated BER for Cameraman $\quad+$ traditional MC-CDMA

Fig. 8. BER vs. Eb/N0 for multi-user environment in AWGN channel with Lena and Cameraman image coded at: (a) 0.125 bpp, (b) $0.25 \mathrm{bpp}$, (c) $0.5 \mathrm{bpp}$, (d) $0.75 \mathrm{bpp}$, (e) $1 \mathrm{bpp}$ (MC-CDMA proposed), and a single user in traditional MC-CDMA [30].

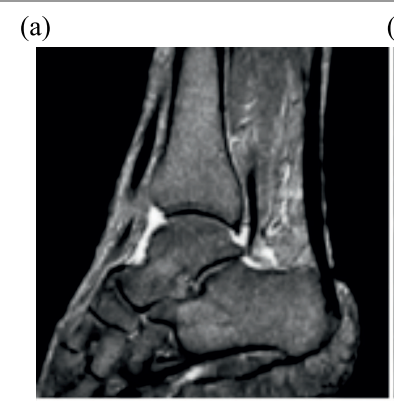

Original image

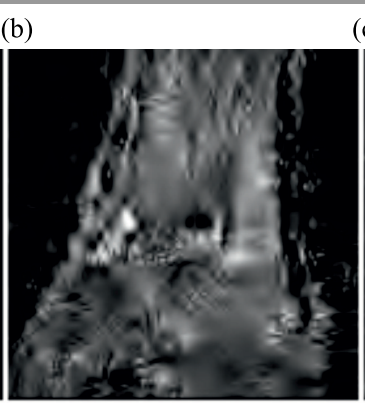

$\mathrm{SNR}=14 \mathrm{~dB}$

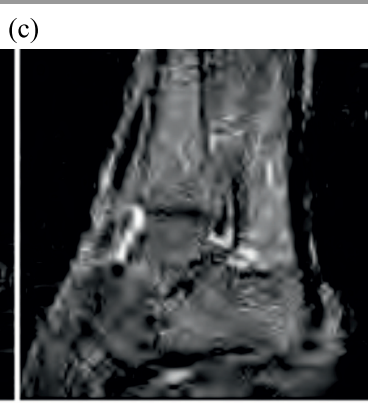

$\mathrm{SNR}=15 \mathrm{~dB}$

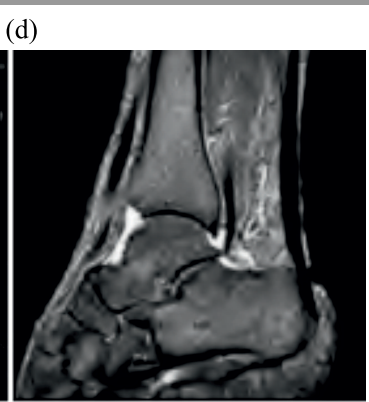

$\mathrm{SNR}=16 \mathrm{~dB}$

Fig. 9. The received IRM1 images with MC-CDMA using $R_{c}=0.75$ bpp over AWGN channel: (a) original image, (b) PSNR = $28.77 \mathrm{~dB}$, (c) PSNR $=29.81 \mathrm{~dB}$, (d) PSNR $=32.67 \mathrm{~dB}$. 


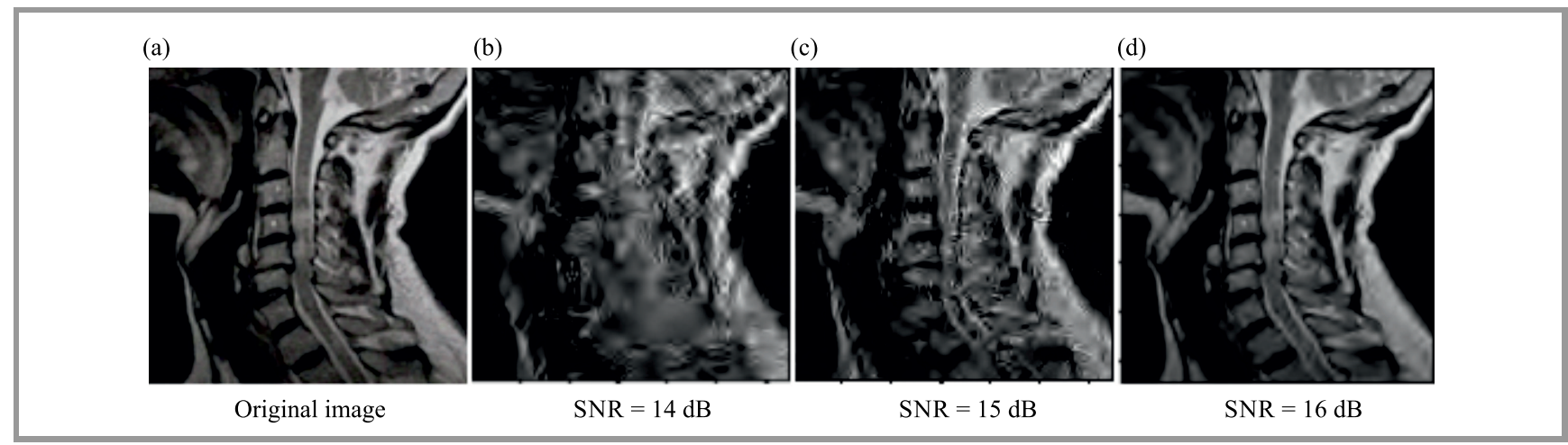

Fig. 10. The received IRM2 images with MC-CDMA using $R_{c}=0.75$ bpp over AWGN channel: (a) original image, (b) PSNR = $28.61 \mathrm{~dB}$, (c) PSNR $=29.35 \mathrm{~dB}$, (d) PSNR $=32.43 \mathrm{~dB}$.

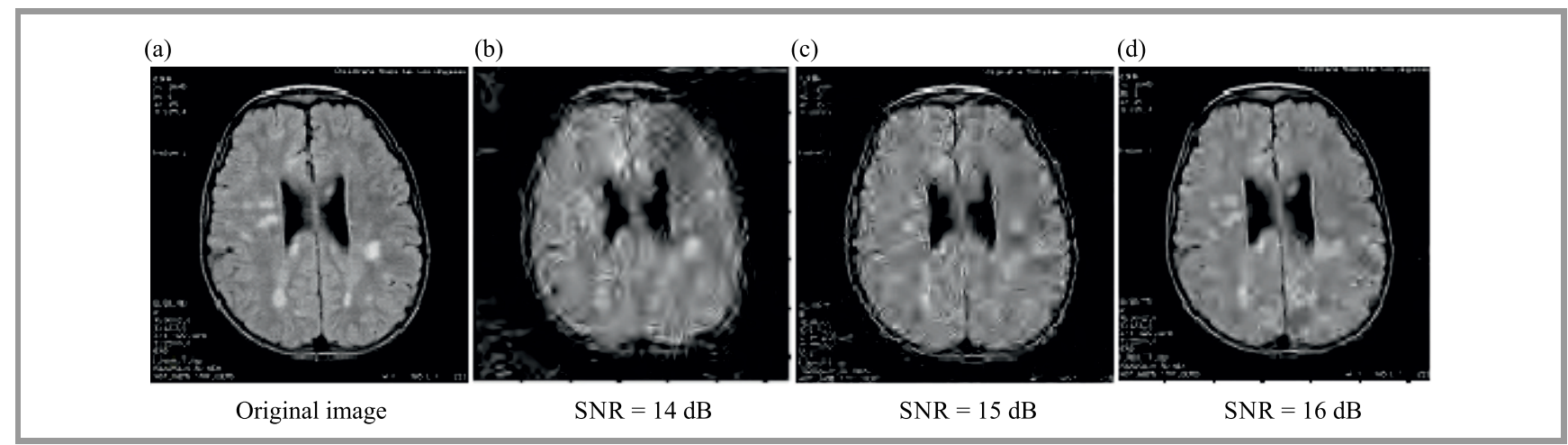

Fig. 11. The received IRM3 images with MC-CDMA using $R_{c}=0.75$ bpp over AWGN channel: (a) original image, (b) PSNR = $29.88 \mathrm{~dB}$, (c) PSNR $=31.87 \mathrm{~dB}$, (d) PSNR $=32.52 \mathrm{~dB}$.

Table 6

Performance results of PSNR and MSSIM values for the IRM1 image at different $R_{c}$ and SNR

\begin{tabular}{|c|c|c|c|c|c|c|c|c|c|c|}
\hline \multirow{2}{*}{$\begin{array}{c}\text { Parameter } \\
\text { of evaluation }\end{array}$} & \multirow{2}{*}{$R_{c}[\mathrm{bpp}]$} & \multirow{2}{*}{$\begin{array}{c}\text { Before } \\
\text { transmission }\end{array}$} & \multicolumn{8}{|c|}{ Transmission with SNR [dB] } \\
\hline & & & 10 & 11 & 12 & 13 & 14 & 15 & 16 & 17 \\
\hline PSNR [dB] & \multirow{2}{*}{0.125} & 29.86 & 27.48 & 27.93 & 28.15 & 28.48 & 29.54 & 29.86 & 29.86 & 29.86 \\
\hline MSSIM & & 0.545 & 0.130 & 0.137 & 0.172 & 0.258 & 0.491 & 0.545 & 0.545 & 0.545 \\
\hline PSNR [dB] & \multirow{2}{*}{0.25} & 30.42 & 27.58 & 28.41 & 28.66 & 28.69 & 28.85 & 30.10 & 30.42 & 30.42 \\
\hline MSSIM & & 0.669 & 0.133 & 0.143 & 0.196 & 0.274 & 0.342 & 0.621 & 0.669 & 0.669 \\
\hline PSNR [dB] & \multirow{2}{*}{0.5} & 31.64 & 26.96 & 27.88 & 28.22 & 28.73 & 28.89 & 29.79 & 31.64 & 31.64 \\
\hline MSSIM & & 0.783 & 0.126 & 0.139 & 0.164 & 0.872 & 0.315 & 0.555 & 0.783 & 0.783 \\
\hline PSNR [dB] & \multirow{2}{*}{0.75} & 32.67 & 27.77 & 27.91 & 28.02 & 28.52 & 28.77 & 29.81 & 32.67 & 32.67 \\
\hline MSSIM & & 0.852 & 0.143 & 0.149 & 0.153 & 0.199 & 0.300 & 0.571 & 0.852 & 0.852 \\
\hline PSNR [dB] & & 33.83 & 27.72 & 28.87 & 28.91 & 29.11 & 29.22 & 29.87 & 33.83 & 33.83 \\
\hline MSSIM & & 0.890 & 0.110 & 0.155 & 0.202 & 0.228 & 0.444 & 0.627 & 0.890 & 0.890 \\
\hline
\end{tabular}

\section{Conclusions}

In this paper, an efficient image transmission system using the downlink MC-CDMA model is presented. The solution proposed transmits images over wireless AWGN channels using DWT associated with the SPIHT coder. According to Shannon's theorem [1], the com- bination of the optimum source coder and optimum channel coder can create an optimum system, therefore joint design of source coder and channel coder is necessary to optimize communication systems [10]. Therefore, the CDF9/7 wavelet is used with the SPIHT coder to optimize data image compression. We can also transmit images with $R_{c}=0.75 \mathrm{bpp}$, which equals to the compression rate $90.63 \%$ of the original image. 
Table 7

Performance results of PSNR and MSSIM values for the IRM2 image at different $R_{c}$ and SNR

\begin{tabular}{|c|c|c|c|c|c|c|c|c|c|c|}
\hline \multirow{2}{*}{$\begin{array}{c}\text { Parameter } \\
\text { of evaluation }\end{array}$} & \multirow{2}{*}{$R_{c}[\mathrm{bpp}]$} & \multirow{2}{*}{$\begin{array}{c}\text { Before } \\
\text { transmission }\end{array}$} & \multicolumn{8}{|c|}{ Transmission with SNR [dB] } \\
\hline & & & 10 & 11 & 12 & 13 & 14 & 15 & 16 & 17 \\
\hline PSNR [dB] & \multirow{2}{*}{0.125} & 29.48 & 27.62 & 27.71 & 27.68 & 28.73 & 29.14 & 29.48 & 29.48 & 29.48 \\
\hline MSSIM & & 0.546 & 0.111 & 0.121 & 0.167 & 0.352 & 0.420 & 0.546 & 0.546 & 0.546 \\
\hline PSNR [dB] & \multirow{2}{*}{0.25} & 30.02 & 27.41 & 28.20 & 28.43 & 28.81 & 29.90 & 30.02 & 30.02 & 30.02 \\
\hline MSSIM & & 0.695 & 0.102 & 0.122 & 0.145 & 0.201 & 0.667 & 0.695 & 0.695 & 0.695 \\
\hline PSNR [dB] & \multirow{2}{*}{0.5} & 31.41 & 27.58 & 28.01 & 28.23 & 28.84 & 29.22 & 30.08 & 31.41 & 31.41 \\
\hline MSSIM & & 0.822 & 0.078 & 0.114 & 0.120 & 0.290 & 0.336 & 0.716 & 0.822 & 0.822 \\
\hline PSNR [dB] & \multirow{2}{*}{0.75} & 32.43 & 27.47 & 27.82 & 27.96 & 28.45 & 28.61 & 29.35 & 32.43 & 32.43 \\
\hline MSSIM & & 0.877 & 0.087 & 0.115 & 0.145 & 0.259 & 0.406 & 0.607 & 0.877 & 0.877 \\
\hline PSNR [dB] & \multirow[t]{2}{*}{1} & 33.59 & 27.54 & 27.66 & 28.57 & 29.07 & 29.60 & 30.49 & 33.45 & 33.59 \\
\hline MSSIM & & 0.912 & 0.093 & 0.110 & 0.136 & 0.220 & 0.306 & 0.753 & 0.906 & 0.912 \\
\hline
\end{tabular}

Table 8

Performance results of PSNR and MSSIM values for the IRM3 image at different $R_{c}$ and SNR

\begin{tabular}{|c|c|c|c|c|c|c|c|c|c|c|}
\hline \multirow{2}{*}{$\begin{array}{c}\text { Parameter } \\
\text { of evaluation }\end{array}$} & \multirow{2}{*}{$R_{c}[\mathrm{bpp}]$} & \multirow{2}{*}{$\begin{array}{c}\text { Before } \\
\text { transmission }\end{array}$} & \multicolumn{8}{|c|}{ Transmission with SNR [dB] } \\
\hline & & & 10 & 11 & 12 & 13 & 14 & 15 & 16 & 17 \\
\hline PSNR [dB] & \multirow{2}{*}{0.125} & 30.13 & 27.75 & 28.41 & 29.56 & 28.48 & 29.80 & 30.13 & 30.13 & 30.13 \\
\hline MSSIM & & 0.527 & 0.148 & 0.169 & 0.320 & 0.258 & 0.464 & 0.527 & 0.527 & 0.527 \\
\hline PSNR [dB] & \multirow{2}{*}{0.25} & 30.59 & 27.39 & 27.87 & 28.35 & 28.69 & 28.61 & 28.87 & 30.59 & 30.59 \\
\hline MSSIM & & 0.630 & 0.142 & 0.167 & 0.208 & 0.274 & 0.231 & 0.343 & 0.630 & 0.630 \\
\hline PSNR $[\mathrm{dB}]$ & \multirow{2}{*}{0.5} & 31.64 & 27.86 & 28.12 & 28.72 & 28.73 & 29.95 & 30.24 & 31.64 & 31.64 \\
\hline MSSIM & & 0.746 & 0.153 & 0.181 & 0.301 & 0.872 & 0.537 & 0.622 & 0.746 & 0.746 \\
\hline PSNR [dB] & \multirow{2}{*}{0.75} & 32.52 & 27.59 & 27.81 & 28.96 & 28.52 & 29.88 & 31.87 & 32.52 & 32.52 \\
\hline MSSIM & & 0.829 & 0.123 & 0.181 & 0.391 & 0.199 & 0.531 & 0.795 & 0.829 & 0.829 \\
\hline PSNR [dB] & \multirow{2}{*}{1} & 34.29 & 27.21 & 27.57 & 29.20 & 29.11 & 29.51 & 30.17 & 34.29 & 34.29 \\
\hline MSSIM & & 0.881 & 0.140 & 0.177 & 0.233 & 0.228 & 0.263 & 0.443 & 0.881 & 0.881 \\
\hline
\end{tabular}

For a perfect reception of the image, we need the SNR = $17 \mathrm{~dB}$ (for natural images). On the other hand, for medical images, the $\mathrm{SNR}=16 \mathrm{~dB}$ is necessary. This enables to use an optimized wireless communications system.

\section{References}

[1] C. E. Shannon, "A mathematical theory of communication", The Bell System Tech. J., vol. 27, no. 3, pp. 379-423, 1948.

[2] C. E. Shannon, "Coding theorems for a discrete source with a fidelity criterion", IRE Int. Conv. Rec., pp. 142-163, 1959.

[3] H. Keang-Po and M. K. Joseph, "Image transmission over noisy channels using multicarrier modulation", Signal Process.: Image Commun., vol. 9, no. 2, pp. 159-169, 1997.

[4] J. Latal et al., "Experimental measurement of thermal turbulence effects on modulated optical beam in the laboratory", in Proc. 19th OptoElectron. and Commun. Conf. and 39th Australian Conf. on Optical Fibre Technol. OECC/ACOFT 2014, Melbourne, Australia, 2014, pp. 455-457.

[5] E. M. El-Bakary et al., "Efficient Image Transmission with Multi-Carrier CDMA", Wireless Pers. Commun., vol. 69, no. 2, pp. 979-994, 2013 (doi: 10.1007/s11277-012-0622-6).
[6] H. Schulze and C. Luders, Theory and Applications of OFDM and CDMA. New York: Wiley, 2005.

[7] K. Fazel and S. Kaiser, Multi-Carrier and Spread Spectrum Systems. Chichester: Wiley, 2003.

[8] S. Hara and R. Prasad, "Overview of multicarrier CDMA", IEEE Commun. Mag., vol. 35, no. 12, pp. 126-133, 1997 (doi: 10.1109/35.642841).

[9] S. Verdu, Multiuser Detection. Cambridge, UK: Cambridge University Press, 1998.

[10] P. P. Dang and P. M. Chau, "Robust image transmission over CDMA channels", IEEE Trans. on Consumer Electron., vol. 46, no. 3, pp. 664-672, 2000.

[11] T. Kathiyaiah and T. H. Oh, "Robust JPEG2000 image transmission through low SNR Multi-Carrier CDMA system in frequencyselective Rayleigh fading channels: a performance study", Int. J. Wavelets Multiresolut Inform. Process., vol. 9, no. 2, pp. 283-303, 2011.

[12] T. H. Oh and G. T. Kim, "Image transmission through MC-CDMA channel: an image quality evaluation", Int. J. Wavelets Multiresolut Inf. Process., vol. 6, no. 6, pp. 827-849, 2008.

[13] K. Ramchandran, M. Vetterli, and C. Herley, "Wavelets subband coding and best bases", Proceedings of the IEEE, vol. 84, no. 4, pp. 541-560, 1996.

[14] R. C. Gonzalez and R. E. Woods, Digital Image Processing. PearsonPrentice Hall, 2005. 
[15] A. S. Vijendran and B. Vidhya, "A hybrid image compression technique using wavelet transformation - MFOCPN and Interpolation", Global J. of Com. Science and Technol., vol. 11, no. 3, pp. 57-62, 2011.

[16] N. Sprljan, S. Grgic, and M. Grgic, "Modified SPIHT algorithm for wavelet packet image coding", Real Time Imag., vol. 11, no. 5-6, pp. 378-388, 2005.

[17] A. Said and A. P. William, "A new, fast, and efficient image codec based on set partitioning in hierarchical trees", IEEE Trans. Circuits and Syst. for Video Technol., vol. 6, no. 3, pp. 243-250, 1996 (doi: 10.1109/76.499834).

[18] A. Bouridane et al., "A very low bit-rate embedded color image coding with SPIHT", in Proc. IEEE Int. Conf. on Acoust., Speech and Sig. Process. ICASSP 2004, Montreal, Quebec, Canada, 2004, pp. 689-692.

[19] J. M. Shapiro, "Embedded image coding using zerotrees of wavelet coefficients", IEEE Trans. on Signal Process., vol. 41, no. 12, pp. 3445-3462, 1993.

[20] I. Boukli-Hacene, M. Beladghem, and A. Bessaid, "Lossy compression color medical image using CDF wavelet lifting scheme", I. J. Image, Graphics and Sig. Process., vol. 11, pp. 53-60, 2013 (doi: 10.5815/ijigsp.2013.11.06).

[21] T. Brahimi, A. Melit, and F. Khelifib, "An improved SPIHT algorithm for lossless image coding”, Digit. Sig. Process., vol. 19, no. 2, pp. 220-228, 2009

[22] S. G. Miaou, S. T. Chen, and S. N. Chao, "Wavelet-based lossyto-lossless medical image compression using dynamic VQ and SPIHT coding", Biomed. Eng. Appl. Basis Commun., vol. 15, no. 6, pp. 235-242, 2003.

[23] Y. Y. Chen and S. C. Tai, "Embedded medical image compression using DCT based subband decomposition and modified SPIHT data organization", in Proc. 4th IEEE Symp. on Bioinform. and Bioengin. BIBE 2004, Taichung, Taiwan, 2004 (doi: 10.1109/BIBE.2004.1317339)

[24] M. Beladgham et al., "MRI image compression using Biorthogonal CDF wavelet based on lifting scheme and SPIHT coding", in Proc. 4th Int. Conf. on Elec. Engin. CIGE'10, Bechar, Algeria, 2010, pp. 225-232, 2010.

[25] Z. Xiong, K. Ramchandran, and M. Orchard, "Space frequency quantization for wavelet image coding", IEEE Trans. on Image Process., vol. 6, no. 5, pp. 677-693, 1997.

[26] K. A. Navas, M. L. Aravind, and M. Sasikumar, "A novel quality measure for information hiding in images", in Proc. IEEE Comp. Society Conf. on Comp. Vision and Pattern Recogn. Worksh. CVPRW'08, Anchorage, AK, USA, 2008 (doi: 10.1109/CVPRW.2008.4562985).

[27] M. Doaa and F. Abou-Chadi, "Image compression using block truncation coding", J. of Selec. Areas in Telecommun. (JSAT), pp. 9-13, 2011.

[28] W. S. Geislerand and M. S. Banks, "Visual performance" in Handbook of Optics, vol. III, M. Bass, Ed. McGraw-Hill, 1995.

[29] A. B. Watson and L. Kreslake, "Measurement of visual impairment scales for digital video", Proc. of SPIE, Human Vision and Electronic Imaging VI, 79, vol. 4299, pp. 79-89, 2001 (doi: 10.1117/12.429526)

[30] M. S. Salih et al., "A proposed improvement model of MCCDMA based FFT in AWGN channel", in IEEE Int. Conf. on Electro/Inform. Technol., Chicago, IL, USA, 2007, pp. 517-520 (doi: 10.1109/EIT.2007.4374460).

[31] S. Nobilet, J.-F Helard, and D. Mottier, "Spreading sequences for uplink and downlink MC-CDMA systems: PAPR and MAI minimization", European Trans. on Telecommun., vol. 13, pp. 465-474, 2002.

[32] K. Fazel and S. Kaiser, Multi-Carrier and Spread Spectrum Systems. New York: Wiley, 2008.

[33] A. Gupta, P. Nigam, and V. Chaurasia, "Removal of cyclic prefix in adaptive OFDM for dynamic spectrum access using DWT and WT", Int. J. of Engin. Sci. \& Res. Technol., vol. 3, no. 3, pp. 1187-1191, 2014.

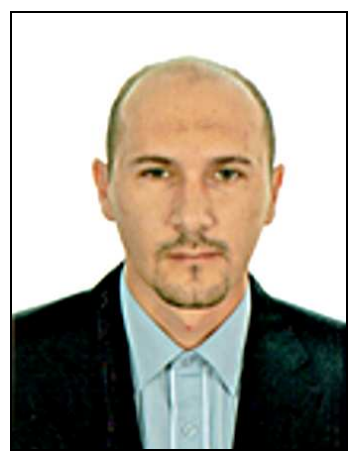

Mohammed Sofiane Bendelhoum received a B.Sc. degree in Electronics Biomedical in 2004, and an M.Sc. in 2008, from Abou Bakr Belkaid University of Tlemcen, Algeria. He is currently pursuing his Ph.D. degree at the Djillali Liabes University of Sidi Bel Abbes, Algeria. His main research interests include the image processing, medical image compression, wavelets transform, wireless communication systems and signal processing.

E-mail: sbendelhoum13@gmail.com

Department of Technologie, Sciences Institute University Center Nour Bachir of El-Bayadh

El-Bayadh, 32000, Algeria

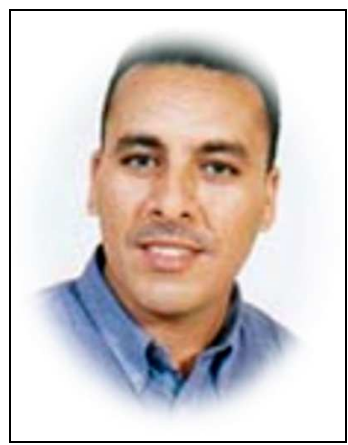

Ali Djebbari received a B.Sc. in 1988, from University of Sciences and Technology of Oran (USTO, Algeria), an M.Sc. in 1991, from Sidi Bel Abbes University, and a Ph.D. in 1997, from USTO. Since 1991, he works at the Department of Telecommunications at the University of Sidi Bel Abbes and performs his research at the Telecommunications and Digital Signal Processing Laboratory. His research interests include signal processing for telecommunications, communication over multipath and fading channels, wireless networks, channel coding for communication networks. He has more than 60 publications in specialized journals with impact factors.

E-mail: adjebari2002@yahoo.fr

Telecommunications and Digital Signal Processing Laboratory Djillali Liabes University

Sidi Bel Abbes, 22000, Algeria

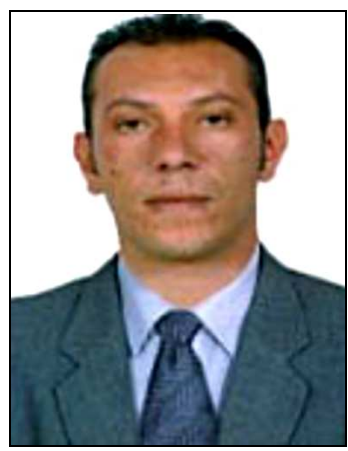

Ismail Boukli-Hacene obtained a B.Sc. in Electronics, an M.Sc. in Electronic Biomedical, and a Ph.D. degree in Electronic Biomedical from University of Tlemcen, Algeria, His research interests are image processing, medical image compression, wavelets transform, wireless communication systems and optimal encoder. He has more than 20 publications in specialized journals with impact factors.

E-mail: ismaill80@yahoo.fr

Biomedical Laboratory

Abou Bekr Belkaid University

Tlemcen, 13000, Algeria 


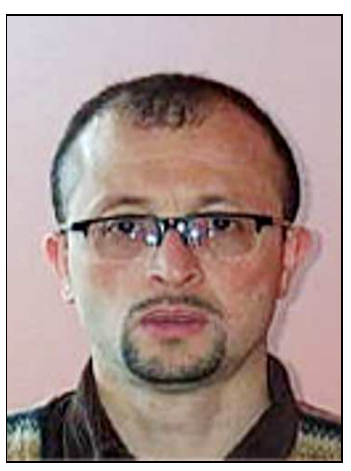

Abdelmalik Taleb-Ahmed received his Ph.D. in Electronics and Microwaves from the University of Lille1 in France in 1992. From 1992 to 2004, he was an Associate Professor at the University of Littoral, Calais. He is currently a Professor at the University of Valenciennes in the department GE2I, and does his research at the
LAMIH FRE CNRS 3304 UVHC. His research interests include signal and image processing, image segmentation, prior knowledge integration in image analysis, partial differential equations and variational methods in image analysis, image compression, multimodal signal processing, medical image analysis, including multimodal image registration. He is an author of more than 200 publications. E-mail: abdelmalik.tale-ahmed@univalenciennes.fr Laboratoire LAMIH UMR CNRS 8530

Valenciennes et du Hainaut Cambrésis University Valenciennes, 59313, France 